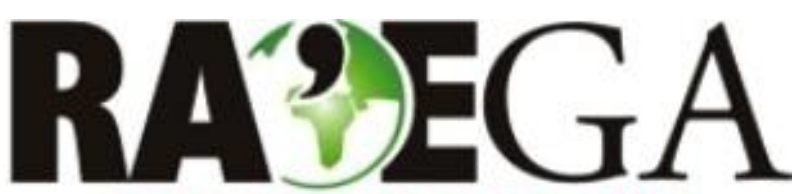

O ESPAÇO GEOGRÁFICO EM ANÁLISE

\title{
ANÁLISE DA SUSCETIBILIDADE DO SOLO A PROCESSOS EROSIVOS DO PARQUE NATURAL MUNICIPAL CORREDORES DE BIODIVERSIDADE (PNMCBIO) DE SOROCABA (SP)
}

\section{SOIL SUSCEPTIBILITY ANALYSIS TO EROSIVE PROCESSES OF THE PARQUE NATURAL MUNICIPAL CORREDORES DE BIODIVERSIDADE (PNMCBIO) OF SOROCABA (SP)}

\author{
Vanessa Cezar Simonetti ${ }^{1 \mathrm{a}}$, Darllan Collins da Cunha e Silva ${ }^{2 \mathrm{~b}}$, Renan Angrizani de Oliveira ${ }^{2 \mathrm{c}}$, Débora \\ Zumkeller Sabonaro ${ }^{2 \mathrm{~d}}$, André Henrique Rosa ${ }^{1 \mathrm{e}}$
}

\section{RESUMO}

Os solos são de fundamental importância para a humanidade, entretanto as atividades antrópicas vêm exercendo forte pressão sobre este recurso, desencadeando processos erosivos. O presente estudo objetivou a identificação das zonas mais suscetíveis à erosão laminar a partir da caracterização dos atributos físico-hídricos do solo em duas zonas distintas do Parque Natural Municipal Corredores de Biodiversidade (PNMCBio) situado no município de Sorocaba (SP). As amostras de solos foram extraídas na Zona Intangível (ZI) e Zona de Recuperação (ZR) na profundidade 0-20 cm, as zonas foram prioritárias para o estudo pelas características distintas e a profundidade ser a mais suscetível à erosão. Em cada zona foram realizadas análises de granulometria, umidade (volumétrica e gravimétrica), densidade aparente e porosidade total, sendo que o fator de erodibilidade do solo (K) foi calculado de forma indireta através da expressão de Bouyoucos a partir dos dados obtidos na análise granulométrica. Esses resultados foram interpolados para análise da distribuição espacial destes atributos na área de estudo. Nas duas zonas, o solo foi classificado como de média erodibilidade, portanto, a manutenção do parque como unidade de conservação garante que o manejo antrópico nesta área seja sustentável e não desencadeia nenhum processo erosivo. O presente estudo traz uma importante contribuição para a caracterização dos atributos físico-hídricos do solo do PNMCBio, possibilitando avaliar de forma espacializada as zonas mais suscetíveis à erosão laminar e servir de suporte para a gestão e tomada de decisão pelos órgãos responsáveis pela sua conservação.

Palavras-chave: erodibilidade do solo; unidades de conservação; erosão hídrica; conservação do solo.

\section{ABSTRACT}

Soils are of fundamental importance for humanity, however anthropic activities have been exerting strong pressure on this resource, triggering erosive processes. The present study aimed to identify the area's most susceptible to laminar erosion from the characterization of soil physical-hydric attributes in two distinct zones of the Parque Natural Municipal Corredores de Biodiversidade (PNMCBio) located in the city of Sorocaba (SP). Soil samples were extracted in the Intangible Zone (ZI) and Recovery Zone (ZR) in depth $0-20 \mathrm{~cm}$, the zones were prioritized for the study by the distinct characteristics and the depth is the most susceptible to erosion. In each zone, analyzes of particle size, moisture (volumetric and gravimetric), apparent density and total porosity were performed, and the soil erodibility factor (K) was calculated indirectly by Bouyoucos expression from the data obtained by the particle size analysis. These results were interpolated to analyze the spatial distribution of these attributes in the study area. In both areas, the soil was classified as medium erodibility, therefore, the maintenance of the park as a conservation unit ensures that the anthropic management in this area is sustainable and does not trigger any erosive process. The present study makes an important contribution to the characterization of the soil physicalhydric attributes in the PNMCBio, making possible to evaluate in a spatialized way the area's most susceptible to laminar erosion and provide support for management and decision-making by the agencies responsible for conservation.

Key-words: soil erodibility; conservation units; hydric erosion; soil conservation.

\footnotetext{
${ }^{1}$ Instituto de Ciência e Tecnologia, Universidade Estadual Paulista, Sorocaba/SP.

${ }^{2}$ Universidade de Sorocaba, Sorocaba/SP.

${ }^{a}$ va_simonetti@hotmail.com, ${ }^{b}$ darllanamb@yahoo.com.br, ${ }^{c}$ renan_angrizani@hotmail.com,

ddzsabonaro@hotmail.com, ${ }^{e}$ ahrosa@sorocaba.unesp.br.
} 
SIMONETTI, V. C., SILVA, D. C. C., OLIVEIRA, R. A., SABONARO, D. Z.,ROSA, A. H.

\section{ANÁLISE DA SUSCETIBILIDADE DO SOLO A PROCESSOS EROSIVOS DO PARQUE NATURAL MUNICIPAL CORREDORES DE BIODIVERSIDADE (PNMCBIO) DE SOROCABA (SP)}

\section{INTRODUÇÃO}

Os solos são de fundamental importância no que tange à qualidade do meio ambiente e beneficiam a humanidade através de diversos serviços ambientais, sendo um recurso fundamental para a produção de alimentos, regulação do ciclo hidrológico, dentre outros (PALM et al., 2007; RAIJ, 2011; PREVEDELLO et al., 2013).

No entanto, às atividades antrópicas resultam em alterações no uso do solo e em suas proporções, exercendo forte pressão nas áreas florestais e também em áreas de ecossistemas não florestais como, por exemplo, os campos (MACEDO et al., 2013). Este processo está relacionado à degradação dos solos, uma vez que a cobertura vegetal atua como uma proteção, amortecendo e interceptando a água da chuva e preservando a sua estrutura, desta forma controlando os processos erosivos (OLIVEIRA et al., 2010; STEFANOSKI et al., 2013).

De acordo com Volk e Cogo (2008), os processos erosivos são determinados pela associação de diversos fatores, dos quais destacase a topografia do terreno, fatores climáticos, uso e manejo do solo, textura e sua erodibilidade intrínseca.

Diante do exposto, o uso de técnicas de geoprocessamento, associados aos Sistemas de Informações Geográficas (SIG), vêm ganhando cada vez mais espaço no cenário atual, sendo uma importante ferramenta de avaliação e monitoramento da paisagem, auxiliando nos processos de planejamento e manejo dos recursos naturais (MELLO et al., 2006; SALES et al., 2016; SILVA et al., 2016).

Vários estudos foram realizados para determinar o fator de erodibilidade em solos de diferentes regiões utilizando o fator $\mathrm{K}$, tais como o de Souza e Galvani (2017), que determinaram o potencial natural à erosão na bacia do rio Jacaré Guaçu no estado de São Paulo, Brasil, utilizando técnicas de geoprocessamento desenvolvidas em
SIG, enquanto que, Arghiuş e Arghiuş (2011) estimaram a taxa anual de erosão superficial do solo nas cordilheiras de Codrului e Piemonte na Romênia utilizando técnicas de SIG para representação espacial, utilizando o modelo de erosão de solo da Romênia. Já Tang et al. (2015), utilizaram a Equação Universal de Perda de Solo Revisada (RUSLE) associada ao emprego de SIG para estimar a perda do solo e sua distribuição espacial da bacia hidrográfica de Yangou na China.

Devido à relevância do conhecimento dos atributos relacionados ao solo, principalmente no que tange aos processos de restauração de áreas degradadas, o presente estudo objetivou a caracterização dos atributos físicos, tais como granulometria, densidade, umidade (volumétrica e gravimétrica) e porosidade na Zona Intangível (ZI) e Zona de Recuperação (ZR) do Parque Natural Corredores de Biodiversidade (PNMCBio). Também foram identificadas as zonas mais suscetíveis à erosão laminar utilizando métodos de determinação indireta (expressão de Bouyoucos), que determina o fator de erodibilidade do solo (fator K) presente na Equação Universal de Perda de Solos (USLE).

\section{MATERIAL E MÉTODOS}

\section{1. ÁREA DE ESTUDO}

Para o desenvolvimento deste estudo foi escolhido o Parque Natural Municipal Corredores de Biodiversidade (PNMCBio), situado entre as coordenadas $245551 \mathrm{~m}$ (W); $247052 \mathrm{~m}$ (E) e $7410377 \mathrm{~m}$ (S); 7411641m (N), ao norte do município de Sorocaba-SP (Figura 1), o qual é uma Unidade de Conservação de Proteção Integral criada pelo Decreto $\mathrm{n}^{\circ} 19.424$, de 17 de agosto de 2011, como parte da medida compensatória proveniente da implantação de uma indústria automobilística no município (SOROCABA, 2012; MOTA JÚNIOR et al., 2015). 


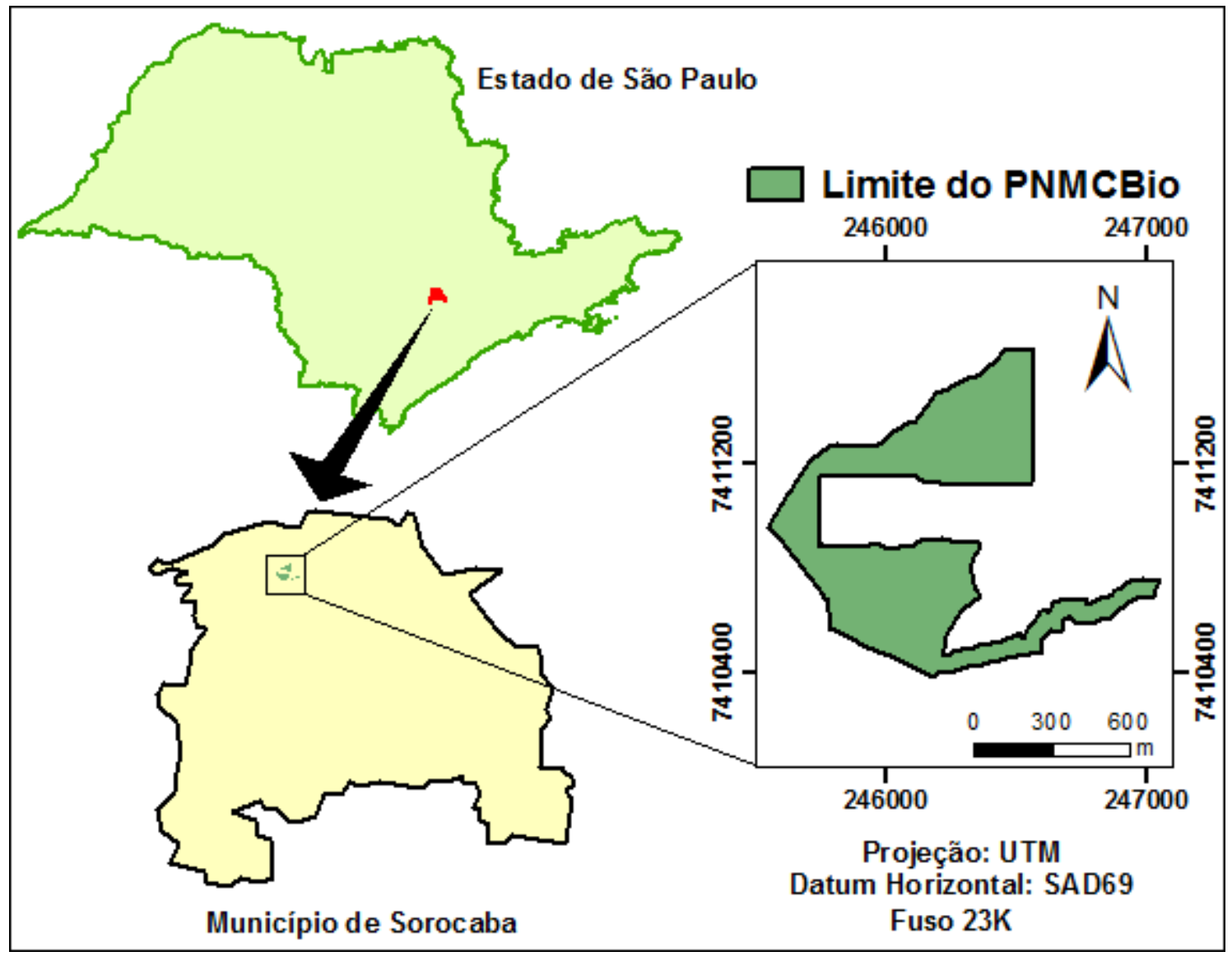

Figura 1 - Localização da área de estudo.

A implantação do parque no município foi tida como prioritária por estar inserido em uma zona de grande aglomeração urbana e diferentes usos e ocupação do solo (OLIVEIRA et al., 2016). A área total do parque é de 62,42 hectares, sendo 31 hectares referentes à área de vegetação nativa, com predominância de Floresta Estacional Semidecidual e Floresta Estacional Semidecidual Aluvial (SOROCABA, 2012).

A classificação climática segundo Köeppen-Geiger é de clima dominante do tipo "Cfa", que caracteriza clima subtropical quente, inverno não muito seco (30 a $60 \mathrm{~mm}$ de precipitação pluviométrica no mês mais seco) tendo como temperatura máxima de verão $30,1^{\circ} \mathrm{C}$ e mínima de inverno $12,2^{\circ} \mathrm{C}$ possuindo uma faixa mais ao norte com características de clima tipo "Cwa", sendo clima temperado chuvoso e quente (com média pluviométrica inferior a $30 \mathrm{~mm}$ no mês mais seco), cuja temperatura média dos meses mais quentes é superior a $22^{\circ} \mathrm{C}$ e inferior a $18^{\circ} \mathrm{C}$ nos meses mais frios. A região possui precipitação média anual é de 1.400 mm (SILVA, 2008; SMITH et al., 2014).

Os solos encontrados na área de estudo, segundo o sistema de classificação de solos da Embrapa (2006), são compostos por Latossolos Vermelhos Distróficos (LVd) A moderado, textura argilosa, relevo suave ondulado e ondulado. Os Latossolos, em geral, são solos minerais, apresentando textura média ou mais fina, variando de argilosa a muito argilosa, homogêneos, possuindo pouca diferenciação entre seus horizontes e camadas, sendo profundos, bem drenados, com elevados teores de $\mathrm{Fe}_{2} \mathrm{O}_{3}$ e, consequentemente, cores mais avermelhadas e com baixa capacidade de troca catiônica. São solos fortemente ácidos, com baixa saturação por bases (OLIVEIRA, 1999; EMBRAPA, 2006; SOROCABA, 2012). 


\section{ANÁLISE DA SUSCETIBILIDADE DO SOLO A PROCESSOS EROSIVOS DO PARQUE NATURAL MUNICIPAL CORREDORES DE BIODIVERSIDADE (PNMCBIO) DE SOROCABA (SP)}

O PNMCBio está inserido na Bacia Sedimentar do Paraná (Depressão Periférica Paulista) e sua geomorfologia se caracteriza como Depressão Médio Tietê com predominância de formas de relevo constituídos em grande parte por colinas de topos amplos tabulares e convexos (SOROCABA, 2012).

Quanto à unidade geológica, apresenta Sedimentos Paleozóicos do Grupo Tubarão, contemplando Sedimentos Predominantemente Arenosos, constituídos de arenitos com granulação variando de fina a média, sendo imaturos e apresentando grãos com diferentes porcentagens de feldspato passando a arcósios em matriz argilosa. Apresentam, também, Sedimentos Predominantemente Argilosos onde predominam os lamitos, com argilitos, ritmitos e arenitos, subordinados. Esses sedimentos pertencem ao Subgrupo Itararé, sendo compostos predominantemente, segundo o mapeamento geológico do município, por arenitos, lamitos e ritmitos bem como associações faciológicas oriundas de ambientes marinhos de águas rasas, de leques deposicionais, pró-deltáico à frente deltáica e de planície deltaica (IG, 1990; SOROCABA, 2012).

\subsection{COLETA DE DADOS IN SITU}

As amostras de solo foram coletadas em duas zonas do PNMCBio (Figura 2), segundo o zoneamento do parque, sendo a Zona Intangível (ZI) e Zona de Recuperação (ZR). Para cada zona foram coletadas 20 subamostras de solo, na profundidade $0-20 \mathrm{~cm}$ por se tratar do perfil com maior suscetibilidade a erosão laminar, sendo um ponto amostral coletado fora do zoneamento denominado $\mathrm{ZI}$ devido às proximidades das áreas e o tênue limite que as separa, sendo de difícil diferenciação

in situ.

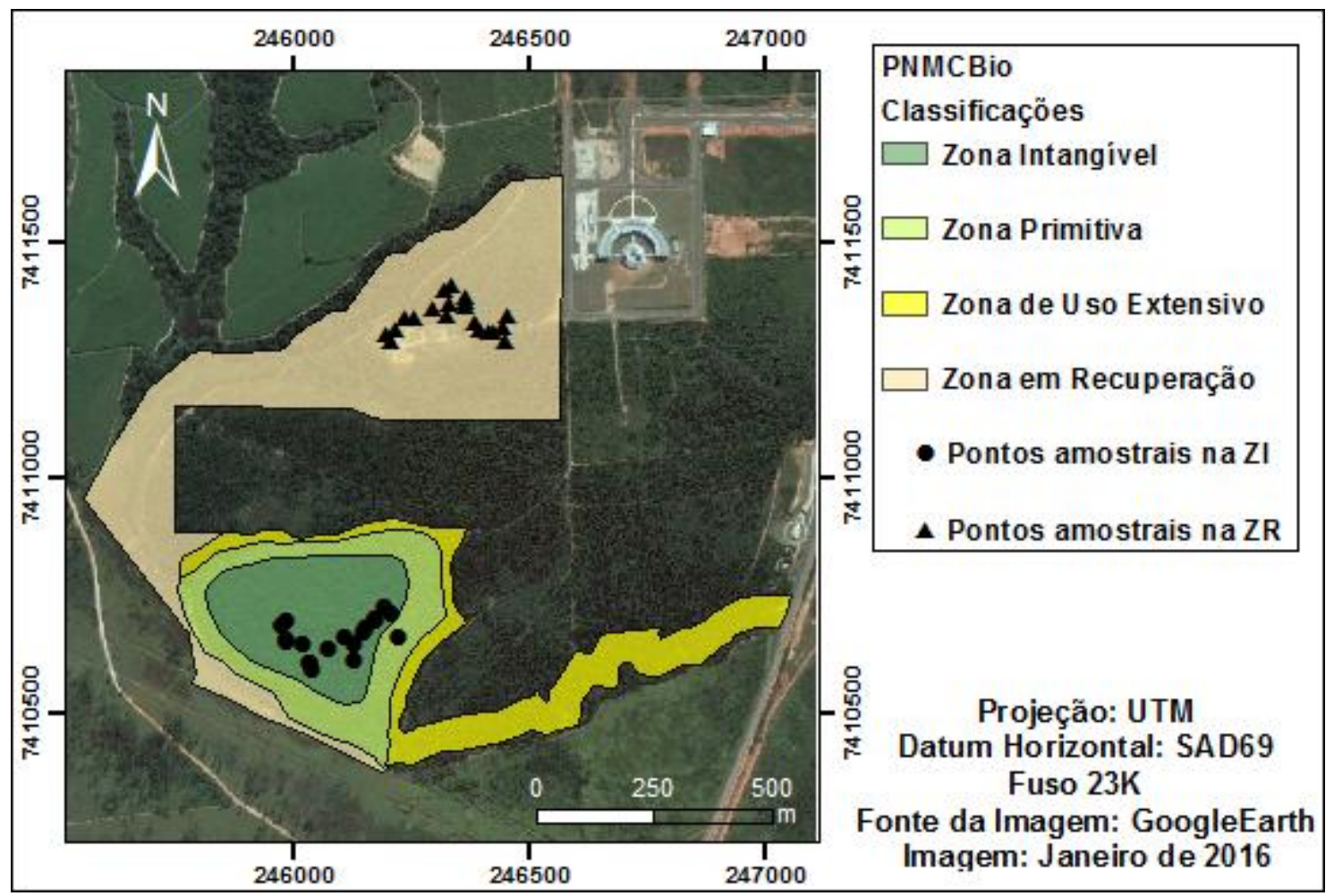

Figura 2 - Localização dos pontos amostrais na área de estudo.

Para a coleta das amostras foi utilizado um trado holandês confeccionado em aço inox, as quais foram homogeneizadas e, desta amostra composta, foram retirados $500 \mathrm{~g}$ e enviadas ao Laboratório de Química Agrícola (S\&F), onde as frações de argila, silte e areia $\left(\mathrm{g} . \mathrm{kg}^{-1}\right)$ foram 


\section{ANÁLISE DA SUSCETIBILIDADE DO SOLO A PROCESSOS EROSIVOS DO PARQUE NATURAL MUNICIPAL CORREDORES DE BIODIVERSIDADE (PNMCBIO) DE SOROCABA (SP)}

mensuradas através do método da pipeta (CAMARGO et al., 2009).

As classificações e os limites apresentados na Figura 2 foram extraídos do mapeamento cartográfico realizado pela empresa Biométrica - Avaliações Biológicas e Manejo Ambiental Ltda. e constam no Plano de Manejo do Parque Natural Municipal Corredores de Biodiversidade de Sorocaba (PNMCBio), enquanto que, a espacialização dos pontos amostrais foi realizada com o auxílio de um Global Position System (GPS) modelo Magellan Triton 400 para a obtenção das coordenadas geográficas.

\subsection{FATOR DE ERODIBILIDADE DO SOLO (K)}

$O$ fator de erodibilidade do solo $(K)$ do PNMCBio foi calculado de forma indireta através da expressão de Bouyoucos (MANNIGEL et al., 2002; BERTONI; LOMBARDI NETO, 2012), que considera apenas a porcentagens das frações de areia, silte e argila, conforme a Equação (1):

$\mathrm{K}=\left(\frac{\% \text { Areia }+\% \text { Silte }}{\% \text { Argila }}\right) \times \frac{1}{100}$

Onde:

$K$ é o fator de erodibilidade do solo (t.ha.h.ha ${ }^{-}$ $\left.{ }^{1} \cdot \mathrm{MJ}^{-1} \cdot \mathrm{mm}^{-1}\right)$;

$\%$ areia, \% silte e \% argila representam as porcentagens das respectivas frações de uma amostra de solo.

O estudo considerou os valores de predisposição à erodibilidade (Tabela 1) propostos por Mannigel et al. (2002) para Latossolos.

Tabela 1 - Classes de erodibilidade para Latossolos.

\begin{tabular}{ll}
\hline Classes & $\begin{array}{l}\text { Fator } \mathrm{K} \\
\text { (t.ha.h.ha }{ }^{-1} \cdot \mathrm{MJ}^{-1} \cdot \mathrm{mm}^{-1} \text { ) }\end{array}$ \\
\hline Extremamente alta & $>0,0600$ \\
Muito alta & 0,0459 a 0,0600 \\
Alta & 0,0300 a 0,0450 \\
Média & 0,0150 a 0,0300 \\
Baixa & 0,0090 a 0,0150 \\
Muito baixa & $<0,0090$ \\
\hline
\end{tabular}

Fonte: Adaptado de Mannigel et al. (2002).

\subsection{DISTRIBUIÇÃO ESPACIAL DE PROPRIEDADES FÍSICO-HÍDRICAS DE DUAS ZONAS DISTINTAS DO PNMCBIO}

A análise da distribuição dos parâmetros pelo PNMCBio foi realizada através do software ArcGis 10.1. A interpolação dos dados coletados em campo foi realizada por meio do interpolador Inverso do Quadrado da Distância (IQD) que é um interpolador univariado de médias ponderadas descrito pela Equação (2). Estudos realizados por Silva et al. (2008) e Souza et al. (2010) concluíram que este interpolador apresenta resultados satisfatórios para estimar a distribuição de atributos físicos dos solos, razão pela qual foi utilizado neste estudo. $x p=\frac{\sum_{i=1}^{n}\left(\frac{1}{d_{i}^{2}} * x i\right)}{\sum_{i=1}^{n}\left(\frac{1}{d_{i}^{2}}\right)}$

Onde: $x p=$ atributo interpolado; $x i=$ valor do atributo do i-ésimo ponto de amostragem; $d i$ = distância euclidiana entre $o i$ ésimo ponto de vizinhança e o ponto amostrado; e $n=$ número de amostras.

Para a caracterização físico-hídrica do solo do PNMCBio foram mensurados os parâmetros concernentes à umidade gravimétrica e volumétrica, densidade aparente e porosidade total, utilizando os anéis de Kopecky com volume de $100 \mathrm{~cm}^{3}$, de acordo com o 


\section{ANÁLISE DA SUSCETIBILIDADE DO SOLO A PROCESSOS EROSIVOS DO PARQUE NATURAL MUNICIPAL CORREDORES DE BIODIVERSIDADE (PNMCBIO) DE SOROCABA (SP)}

método do anel volumétrico proposto pela Embrapa (1997) e Camargo et al. (2009).

As amostras foram coletadas nas ZI e ZR por apresentarem características distintas que poderiam refletir significativamente nos resultados provenientes dos diferentes manejos às quais foram submetidas, sendo a ZI a área mais preservada do PNMCBio e a ZR a área com maior interferência antrópica. Após coletadas, as amostras foram transportadas sob condições adequadas ao Núcleo de Estudos Ambientais (NEAS) da Universidade de Sorocaba (Uniso), onde foram mensuradas.

A porosidade total foi estimada segundo a Embrapa (1997), na qual utiliza-se os valores de densidade aparente e densidade de partículas, conforme a Equação (3):

Porosidade total $=100 \times \frac{(\alpha-\beta)}{\alpha}$

Onde:

$\alpha$ é a densidade de partículas

$\beta$ é a densidade aparente

\section{RESULTADOS E DISCUSSÃO}

A Tabela 2 apresenta as características granulométricas das zonas estudadas, podendo ser observado que ambas as zonas apresentam teores de argila semelhantes, diferenciando-se bastante com relação ao teor de areia fina e silte, contudo a classificação textural de ambas as zonas é franco argiloso.

Tabela 2 - Características granulométricas $\left(\mathrm{g} . \mathrm{kg}^{-1}\right)$ das zonas estudadas.

\begin{tabular}{rccccc}
\hline Zonas $^{1}$ & Areia Grossa & Areia fina & Silte & Argila & Classificação Textural \\
\hline ZI & 24 & 352 & 312 & 312 & Franco Argiloso \\
ZR & 20 & 195 & 465 & 320 & Franco Argiloso \\
\hline \multicolumn{5}{r}{ (1) ZI - Zona Intangível, ZR - Zona de Recuperação. }
\end{tabular}

A partir dos resultados das análises granulométricas (Tabela 2), foi possível determinar o valor do fator de erodibilidade do solo para cada uma das zonas estudadas.

Os valores de erodibilidade foram apresentados na Figura 3, sendo que a Zona
Primitiva e a Zona de Uso Extensivo foram agregadas à Zona Intangível devido à proximidade para a representação dos valores de erodibilidade

do

solo. 
SIMONETTI, V. C., SILVA, D. C. C., OLIVEIRA, R. A., SABONARO, D. Z.,ROSA, A. H.

\section{ANÁLISE DA SUSCETIBILIDADE DO SOLO A PROCESSOS EROSIVOS DO PARQUE NATURAL MUNICIPAL CORREDORES DE BIODIVERSIDADE (PNMCBIO) DE SOROCABA (SP)}

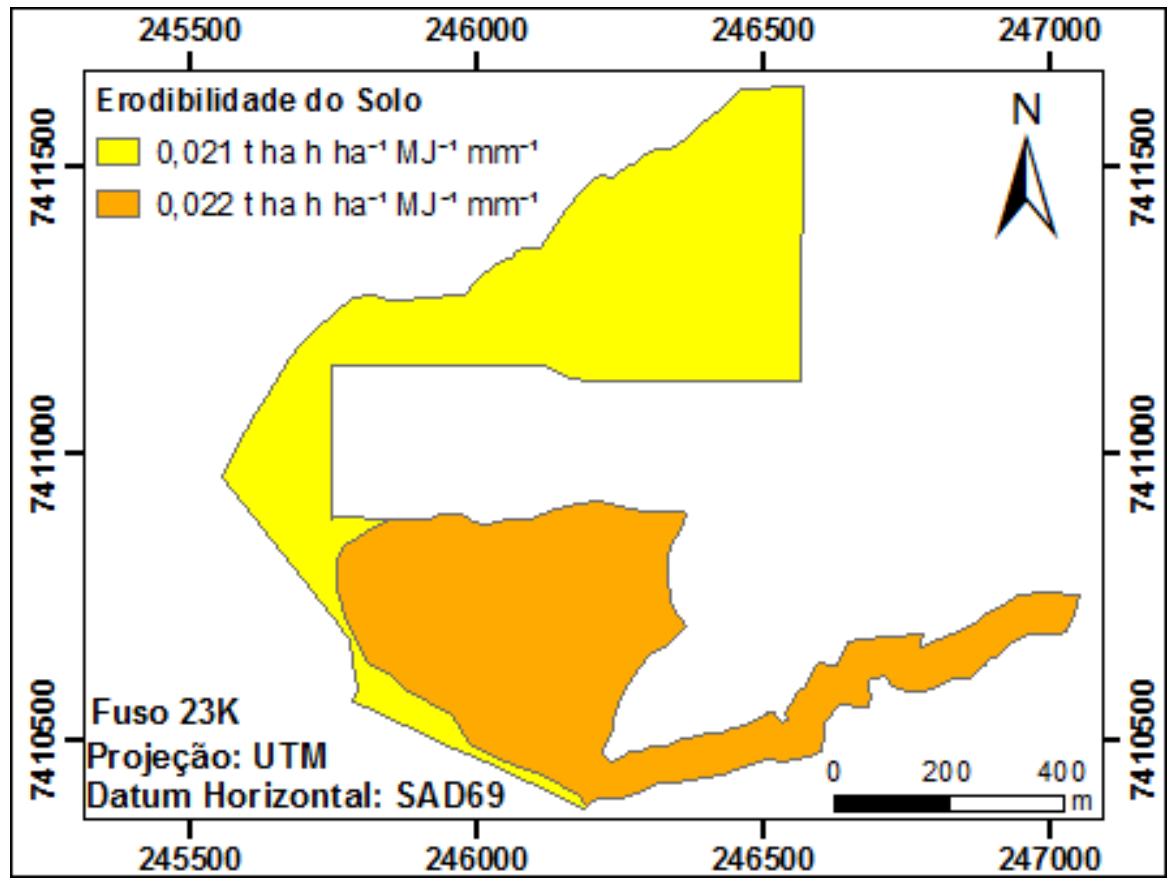

Figura 3 - Distribuição dos valores de erodibilidade do solo para o PNMCBio.

Devido as dimensões do PNMCBio e da proximidade das ZI e ZR, os valores de erodibilidade do solo apresentaram baixa variabilidade espacial. No entanto, a área que contempla a ZR apresentou um valor de erodibilidade de 0,021 t.ha.h.ha ${ }^{-1} \cdot \mathrm{MJ}^{-1} \cdot \mathrm{mm}^{-1}$, sendo 0,001 t.ha.h.ha ${ }^{-1} \cdot \mathrm{MJ}^{-1} \cdot \mathrm{mm}^{-1}$ inferior à área adjacente a qual está inserida a Zl $(0,022$ t.ha.h.ha ${ }^{-1} \cdot \mathrm{MJ}^{-1} \cdot \mathrm{mm}^{-1}$ ). Esses resultados conferem ao PNMCBIO, segundo Mannigel et al. (2002) a classificação de média erodibilidade.

O estudo realizado por Freire e Pessotti (1974) revela que a erodibilidade do solo do estado de São Paulo varia de 0,02 a 0,67 t.ha.h.ha ${ }^{-1} \cdot \mathrm{MJ}^{-1} \cdot \mathrm{mm}^{-1}$, valores que corroboram com os resultados obtidos no estudo. Nesse sentido, Silva (2008), analisando o potencial de erosão laminar no município de Sorocaba, também encontrou uma erodibilidade variando de média a alta para os solos do município. Todavia, Silva e Alvares (2005) compilaram dados de diversas pesquisas existentes sobre erodibilidade do solo no estado de São Paulo, obtendo o valor médio de $K$ igual a 0,0162 t.ha.h.ha ${ }^{-1} \cdot \mathrm{MJ}^{-1} \cdot \mathrm{mm}^{-1}$ para a classe de Latossolos.
Embora a Zona de Recuperação tenha apresentado menor valor de erodibilidade se comparada à Zona Intangível, as campanhas de coletas de dados in situ revelaram diversas feições erosivas na ZR.

Nesse sentido, entende-se que o uso do solo sem a execução de práticas conservacionistas e manejo adequado desencadearam diversos processos erosivos resultantes do cultivo de monocultura que havia na área. Essas feições erosivas também podem se agravar em razão das chuvas locais, declividade, falta de cobertura vegetal, além das características intrínsecas dos solos (STEFANOSKI et al., 2013).

Entretanto, de modo a aumentar a acurácia do estudo de erodibilidade do PNMCBio, também foram analisados os atributos físicos (Figura 4), classificados por Doran e Parkin (1994) como passíveis de sofrerem alterações de acordo com o sistema de manejo do solo. Ainda, segundo os autores esses atributos físicos são amplamente utilizados como indicadores de qualidade física do solo. 


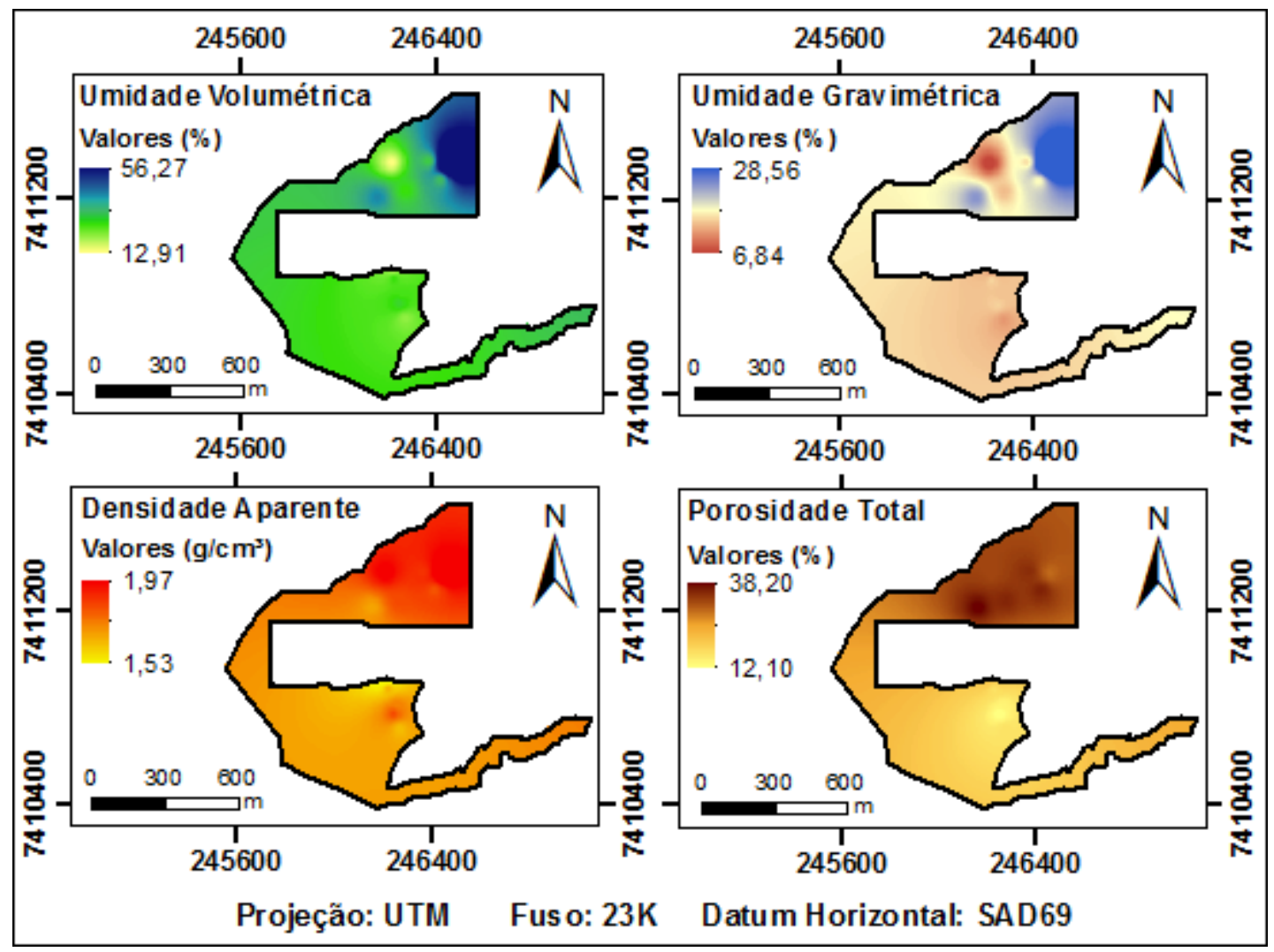

Figura 4 - Distribuição dos parâmetros físico-hídricos do solo para o PNMCBio.

Os resultados demonstraram que todos os parâmetros físico-hídricos analisados apresentaram uma peculiaridade na sua distribuição (Figura 4), pois todos demonstraram altos valores na Zona de Recuperação (ZR). No entanto, esses resultados evidenciaram que às atividades decorrentes de práticas de monoculturas que havia na ZR alteraram significativamente os atributos físicos referentes à umidade volumétrica e gravimétrica, densidade aparente e porosidade.

A razão entre o valor máximo e mínimo é mais acentuada para o parâmetro de umidade volumétrica, enquanto que a razão, para o parâmetro de densidade aparente é menor. Os valores mais acentuados de densidade na Zona de Recuperação podem ser atribuídos aos impactos provenientes de atividades de monocultura, como o plantio de Eucalyptus e Pinus que havia na área. Já os menores valores de densidade da $\mathrm{ZI}$ podem ser decorrentes da cobertura vegetal presente na área, visto que essa é a área do parque que apresenta remanescentes florestais de Floresta Estacional Semidecidual em melhor estado de preservação (SOROCABA, 2012).

De acordo com Stefanoski et al. (2013) a vegetação nativa possui um importante papel na preservação da estrutura do solo, pois favorece a integridade de características estruturais, como densidade, porosidade e permeabilidade do solo. Ainda, protege o solo contra a erosão devido à deposição de serapilheira, diminuindo o impacto das gotas de chuva no solo, além da interceptação da água pelas copas das árvores (OLIVEIRA et al., 2010).

Os valores obtidos, se comparados a outros estudos, como o de Stone e Silveira (2001) apresentaram valores semelhantes para densidade aparente, porém valores inferiores para porosidade total na ordem de $10 \%$ a menos do valor máximo, enquanto que, para umidade volumétrica está dentro da média quando comparado com o estudo realizado por Imhoff et 
SIMONETTI, V. C., SILVA, D. C. C., OLIVEIRA, R. A., SABONARO, D. Z.,ROSA, A. H.

\section{ANÁLISE DA SUSCETIBILIDADE DO SOLO A PROCESSOS EROSIVOS DO PARQUE NATURAL MUNICIPAL CORREDORES DE BIODIVERSIDADE (PNMCBIO) DE SOROCABA (SP)}

al. (2000). Ao analisar a umidade gravimétrica com o estudo realizado por Maria et al. (1999), constatou-se que os valores encontrados para o PNMCBio estão, em sua maioria, abaixo dos valores encontrados por esses pesquisadores.

No entanto, Bono et al. (2013) alertam que para uma avaliação mais conclusiva sobre o estado de conservação do solo seja realizado um acompanhamento temporal, uma vez que a sazonalidade pode alterar os atributos físicos relacionados à umidade e porosidade.

De acordo com Stefanoski et al. (2013), fatores como a umidade, tipo e teor de argila, mineralogia, propriedades químicas, biológicas entre outras substâncias orgânicas e inorgânicas podem afetar a resistência dos agregados bem como sua friabilidade.

Nesse sentido, o conhecimento de tais atributos pode nortear as necessidades de intervenção nas áreas do parque que estão sendo recuperadas por meio do plantio de espécies nativas, podendo inferir na necessidade de irrigação destas áreas; sendo assim, os parâmetros físico-hídricos analisados (Figura 4) são de fundamental importância para o estudo dos solos, pois indicam em que condições hídricas encontram-se os mesmos.

Portanto, a partir dos resultados obtidos no estudo levando em consideração o Fator (K) expresso pela equação de Bouyoucos é possível afirmar que o solo do PNMCBio apresenta média erodibilidade.

Os estudos complementares físicoshídricos, tais como à umidade volumétrica e gravimétrica, densidade aparente e porosidade, auxiliaram de modo geral no diagnóstico físico do solo do parque, tendo em vista que esses atributos também podem interferir nos processos de erodibilidade, pois influenciam nas características estruturais do solo.

Contudo, os aspectos geomorfológicos e a unidade pedológica da área de estudo também podem influenciar no desencadeamento de processos erosivos, bem como o uso e ocupação do solo e as práticas de manejo adotadas no entorno desta unidade de conservação.

Portanto, o conhecimento da dinâmica dos potenciais processos erosivos do parque é fundamental para o entendimento dos fatores que podem desencadear esses processos, norteando a utilização das ferramentas e instrumentos de gestão que mais se adequam a necessidade de manutenção e conservação do parque.

\section{CONCLUSÕES}

As zonas estudadas apresentaram valores físico-hídricos aquém do recomendado para um bom crescimento e desenvolvimento das plantas. Fatores como umidade, porosidade e densidade influenciam na distribuição dos nutrientes na composição do solo e consequente crescimento das raízes.

Os resultados obtidos no estudo demonstram que os valores de umidade e porosidade foram mais satisfatórios na Zona de Recuperação, contudo, a densidade aparente apresentou valores superiores se comparados à Zona Intangível, o que sugere que o uso que havia na área com o sistema de monocultura, influenciou na capacidade físico-hídrica do solo. Os resultados também revelaram que o fragmento de Floresta Estacional Semidecidual presente na Zona Intangível afetaram positivamente a área em relação à densidade do solo.

Com relação ao fator $\mathrm{K}$ foi verificado que o solo do PNMCBio é classificado como de média erodibilidade, portanto, a manutenção do parque como unidade de conservação garante que o manejo antrópico nesta área seja sustentável e não desencadeia nenhum processo erosivo.

Todavia, o presente estudo confere um importante ponto de partida no que tange ao conhecimento dos atributos físico-hídricos e erodibilidade do solo do PNMCBio, fornecendo dados para o diagnóstico inicial do seu estado de conservação, e subsídios para a atualização do Plano de Manejo e gestão da Unidade de Conservação. 
SIMONETTI, V. C., SILVA, D. C. C., OLIVEIRA, R. A., SABONARO, D. Z.,ROSA, A. H.

\section{ANÁLISE DA SUSCETIBILIDADE DO SOLO A PROCESSOS EROSIVOS DO PARQUE NATURAL MUNICIPAL CORREDORES DE BIODIVERSIDADE (PNMCBIO) DE SOROCABA (SP)}

\section{REFERÊNCIAS}

ARGHIUŞ, C.; ARGHIUŞ, V. The quantitative estimation of the soil erosion using USLE type ROMSEM model: Case-study-the Codrului ridge and Piedmont (Romania). Carpathian Journal of Earth Environmental Sciences, v. 6, n. 2, p. 59-66, $2011 . \quad$ Disponível em: http://www.ubm.ro/sites/CJEES/

viewTopic.php?topicld=156. Acesso em: 14, julho, 2017.

BERTONI, J.; LOMBARDI NETO, F. Conservação do Solo. 8ed. São Paulo: Ícone, 2012.

BONO, J.A.M.; MACEDO, M.C.M.; TORMENA, C.A. Qualidade física do solo em um Latossolo Vermelho da região sudoeste dos cerrados sob diferentes sistemas de uso e manejo. Revista Brasileira de Ciência do Solo, v. 37, n. 3, p. 743753, 2013. Disponível em: http://www.sbcs.org.br/wp-

content/uploads/2013/07/V37N3a21.pdf. Acesso em: 25, janeiro, 2015.

CAMARGO, O.A.; MONIZ, A.C.; JORGE, J.A.; VALADARES, J.M.A.S. Métodos de Análise Química, Mineralógica e Física de Solos do Instituto Agronômico de Campinas. Campinas: Instituto Agronômico/Fundação IAC, 2009. (Boletim técnico, 106). Disponível em: http://www.iac.sp.gov.br/publicacoes/porassunt o/pdf/Boletim106.pdf. Acesso em: 10, janeiro, 2015.

DORAN, J.W.; PARKIN, T.B. Defining and assessing soil quality. In DORAN, J.W.; CELEMAN, D.C.; BEZDICEK, D.F.; STEWART, B.A. (Org.) Defining soil quality for sustainable environment. Madison: Soil Science Society of America, 1994. p. 3-21.

EMBRAPA - EMPRESA BRASILEIRA DE PESQUISA AGROPECUÁRIA. Centro Nacional de Pesquisa de Solos. Sistema brasileiro de classificação de solos. 2ed. Rio de Janeiro, 2006. Disponível em: https://www.infoteca.cnptia.embrapa.br/infotec a/bitstream/doc/338818/1/sistemabrasileirodecl assificacaodossolos2006.pdf. Acesso em: 17, julho, 2017.

EMBRAPA - EMPRESA BRASILEIRA DE PESQUISA AGROPECUÁRIA. Centro Nacional de Pesquisa de Solos. Manual de métodos de análise de solo. 2ed. Rio de Janeiro, 1997. Disponível em: https://www.agencia.cnptia.embrapa.br/Reposit orio/Manual+de+Metodos_000fzvhotqk02wx5ok 0q43aOram31wtr.pdf. Acesso em: 10, março, 2015.

FREIRE, O.; PESSOTTI, J.E.S. Erodibilidade dos solos do Estado de São Paulo. In: Anais da Escola Superior de Agricultura Luiz de Queiroz. Piracicaba, v. 33, p. 333-350, 1974. Disponível em:

http://www.revistas.usp.br/aesalq/article/viewFil e/39045/41929. Acesso em: 12, janeiro, 2015.

IG - INSTITUTO GEOLÓGICO. Subsídios do meio físico-geológico ao planejamento do Município de Sorocaba (SP). São Paulo: Instituto Geológico (SP), 1990. (Relatório Técnico). Disponível em: http://www.igeologico.sp.gov.br/pc_prodcient_r esult.asp?offset=1230. Acesso em: 16, julho, 2017.

IMHOFF, S.; SILVA, Á.P.; TORMENA, C.A. Aplicações da curva de resistência no controle da qualidade física de um solo sob pastagem. Pesquisa Agropecuária Brasileira, v. 35, n. 7, p. 1493-1500, 2000. Disponível em: http://www.scielo.br/pdf/\%0D/pab/v35n7/1493. pdf. Acesso em: 16, janeiro, 2015.

MACEDO, R.C.; ALMEIDA, C.M.; SANTOS, J.R.; RUDORFF, B.F.T. Modelagem dinâmica espacial das alterações de cobertura e uso da terra relacionadas à expansão canavieira. Boletim de Ciências Geodésicas, v. 19, n. 2, p. 313-337, 2013. Disponível em: http://www.scielo.br/pdf/bcg/v19n2/a09v19n2. Acesso em: 18, julho, 2017.

MANNIGEL, A.R.; PASSOS, M.; MORETI, D.; ROSA MEDEIROS, L. Fator erodibilidade e tolerância de perda dos solos do Estado de São Paulo. Acta Scientiarum Agronomy, v. 24, n. 5, p. 1335-1340, 
SIMONETTI, V. C., SILVA, D. C. C., OLIVEIRA, R. A., SABONARO, D. Z.,ROSA, A. H.

\section{ANÁLISE DA SUSCETIBILIDADE DO SOLO A PROCESSOS EROSIVOS DO PARQUE NATURAL MUNICIPAL CORREDORES DE BIODIVERSIDADE (PNMCBIO) DE SOROCABA (SP)}

2002.

Disponível

em:

http://periodicos.uem.br/ojs/index.php/ActaSciA gron/article/view/2374. Acesso em 16, janeiro, 2015.

MARIA, I.C.; CASTRO, O.M.; SOUZA DIAS, H. Atributos físicos do solo e crescimento radicular de soja em Latossolo Roxo sob diferentes métodos de preparo do solo. Revista Brasileira de Ciência do Solo, v. 23, n. 3, p. 703-709, 1999. Disponível em: http://dx.doi.org/10.1590/S010006831999000300025 . Acesso em 05, março, 2015.

MELLO, G.; BUENO, C.; PEREIRA, G. Variabilidade espacial de perdas de solo, do potencial natural e risco de erosão em áreas intensamente cultivadas. Revista Brasileira de Engenharia Agrícola e Ambiental, v. 10, p. 315-322, 2006. Disponível em: http://dx.doi.org/10.1590/S141543662006000200010. Acesso em: 02, fevereiro, 2015.

MOTA JUNIOR, V.D.; AMORIM, S.R.; GRAHN, M.L.P.; ZAMPIERI, L.Q.R.; SMITH, W.S. Histórico e criação do Parque Natural Municipal Corredores de Biodiversidade. In: SMITH, W.S. e RIBEIRO, C.A. (Org.). Parque Natural Municipal Corredores de Biodiversidade: pesquisas e perspectivas futuras. Sorocaba: Prefeitura Municipal, Secretaria do Meio Ambiente, 2015. p. 16 - 20.

OLIVEIRA, C.A.; KLIEMANN, H.J.; CORRECHEL, V.; SANTOS, F.C.V. Avaliação da retenção de sedimentos pela vegetação ripária pela caracterização morfológica e físico-química do solo. Revista Brasileira de Engenharia Agrícola e Ambiental, v. 14, n. 12, p. 1281-1287, 2010. Disponível em: http://dx.doi.org/10.1590/S141543662010001200005. Acesso em 17, julho, 2017.

OLIVEIRA, J.B. Solos do Estado de São Paulo: descrição das classes registradas no mapa pedológico. Campinas: Instituto Agronômico de Campinas, 1999. 112p. (Boletim Científico, 45.)

OLIVEIRA, R.A.; SILVA, D.C.C.; SIMONETTI, V.C.; STROKA, E.A.B.; SABONARO, D.Z. Proposição de Corredor Ecológico entre duas Unidades de
Conservação na Região Metropolitana de Sorocaba. Revista do Departamento de Geografia, v. 32, p. 61-71, 2016. Disponível em: http://dx.doi.org/10.11606/rdg.v32i0.116467. Acesso em: 14, julho, 2017.

PALM, C.; SANCHEZ, P.; AHAMED, S.; AWITI, A. Soils: A Contemporary Perspective. The Annual Review of Environment and Resources, New York, v. 32, p. 99-129, 2007. Disponível em: https://doi.org/10.1146/annurev.energy.31.0201 05.100307. Acesso em: 04, março, 2015.

PREVEDELLO, J.; VOGELMANN, E.S.; KAISER, D.R.; REINERT, D.J. A funcionalidade do sistema poroso do solo em floresta de eucalipto sob Argissolo. Scientia Forestalis, v. 41, n. 100, p. 557-566, $2013 . \quad$ Disponível em: http://www.ipef.br/publicacoes/scientia/nr100/c ap13.pdf. Acesso em: 05, janeiro, 2015.

RAIJ, B.V. Fertilidade do solo e manejo de nutrientes. Piracicaba: International Plant Nutrition Institute, 2011.

SALES, J.C.A.; SILVA, D.C.C.; ROMAGNANO, L.F.T.; BERTAGNA, R.; LOURENÇO, R.W. Avaliação do impacto ambiental causado pelas alterações espaço temporal do uso do solo e da cobertura vegetal utilizando o modelo das cadeias de markov. Ciência e Natura, v. 38, n. 1, p. 115-124, 2016. Disponível em: http://dx.doi.org/10.5902/2179460X18641. Acesso em: 14, julho, 2017.

SILVA, A.M.; ALVARES, C.A. Erodibilidade dos solos paulistas: levantamento de informações e estruturação de um banco de dados. Geociências, v. 24, n. 1, p. 33-42, 2005. Disponível em: http://www.revistageociencias.com.br/24_1/Art \%203.pdf. Acesso em: 11, fevereiro, 2015.

SILVA, A.M. Potencial natural de erosão no município de Sorocaba, São Paulo, Brasil. Revista Internacional de Desastres Naturales, Accidentes e Infraestructura Civil, v. 8, p. 5-14, 2008. Disponível em: http://academic.uprm.edu/laccei/index.php/RID 
SIMONETTI, V. C., SILVA, D. C. C., OLIVEIRA, R. A., SABONARO, D. Z.,ROSA, A. H.

\section{ANÁLISE DA SUSCETIBILIDADE DO SOLO A PROCESSOS EROSIVOS DO PARQUE NATURAL MUNICIPAL CORREDORES DE BIODIVERSIDADE (PNMCBIO) DE SOROCABA (SP)}

NAIC/article/viewFile/146/142. Acesso em 07, janeiro, 2015.

SILVA, D.C.C.; ALBUQUERQUE FILHO, J.L.; SALES, J.C.A.; LOURENÇO, R.W. Use of morphometric indicators as tools for assessment watershed. Revista Brasileira de Geografia Física, v. 9, p. 627642, 2016. Disponível em: http://dx.doi.org/10.5935/1984-2295.20160043. Acesso em, 15, julho, 2017.

SILVA, S.D.A.; SOUZA LIMA, J.S.D.; SOUZA, G.S.D.; OLIVEIRA, R.B.D. Avaliação de interpoladores estatísticos e determinísticos na estimativa de atributos do solo em agricultura de precisão. Idesia (Arica), v. 26, n. 2, p. 75-81, 2008. Disponível em: http://dx.doi.org/10.4067/S071834292008000200010. Acesso em: 12, janeiro, 2015.

SMITH, W. S.; MOTA JUNIOR, V. D.; CARVALHO, J. L. Biodiversidade do município de Sorocaba. Sorocaba: Prefeitura Municipal de Sorocaba, Secretaria do Meio Ambiente, 2014. 272 p.

SOROCABA. Secretaria do Meio Ambiente. Plano de Manejo do Parque Natural Municipal Corredores de Biodiversidade de Sorocaba. Sorocaba: Biométrica, 2012. Disponível em: http://meioambiente.sorocaba.sp.gov.br/gestaoa mbiental/plano-de-manejo-do-parque-dabiodiversidade/. Acesso em: 15, julho, 2017.

SOUZA, G.D.; LIMA, J.D.S.; XAVIER, A.C.; ROCHA, W.D. Krigagem ordinária e inverso do quadrado da distância aplicados na espacialização de atributos químicos de um argissolo. Scientia Agraria, v. 11, n. 1, p. 73-81, 2010. Disponível em: http://dx.doi.org/10.5380/rsa.v11i1.15939.

Acesso em: 04, janeiro, 2015.
SOUZA, V.; GALVANI, E. Determinação do potencial natural à erosão laminar na Bacia do Rio Jacaré Guaçú (SP). RA'E GA-O Espaço Geográfico em Análise, v. 39, p. 08-23, 2017. Disponível em: http://revistas.ufpr.br/raega/article/view/40661/ 32062. Acesso em: 14, julho, 2017.

STEFANOSKI, D.C., SANTOS, G.G., MARCHÃO, R.L., PETTER, F.A.; PACHECO, L.P. Uso e manejo do solo e seus impactos sobre a qualidade física. Revista Brasileira de Engenharia Agrícola e Ambiental, v. 17, n. 12, 1301-1309, 2013. Disponível em: http://dx.doi.org/10.1590/S141543662013001200008. Acesso em: 15, julho, 2017.

STONE, L.F.; SILVEIRA, P.M. Efeitos do sistema de preparo e da rotação de culturas na porosidade e densidade do solo. Revista Brasileira de Ciência do Solo, v. 25, n. 2, p. 395-401, 2001. Disponível em: http://dx.doi.org/10.1590/S010006832001000200015. Acesso em: 13, janeiro, 2015.

TANG, Q.; XU, Y.; BENNETT, S.J.; LI, Y. Assessment of soil erosion using RUSLE and GIS: a case study of the Yangou watershed in the Loess Plateau, China. Environmental Earth Sciences, v. 73, n. 4, p. 1715-1724, 2015. Disponível em: http://dx.doi.org/10.1007/s12665-014-3523-z. Acesso em: 14, julho, 2017.

VOLK, L.B.S.; COGO, N.P. Inter-relação biomassa vegetal subterrânea-estabilidade de agregadoserosão hídrica em solo submetido a diferentes formas de manejo. Revista Brasileira de Ciência do Solo, v. 32, n. 4, p. 1713-1722, 2008. Disponível em: http://www.scielo.br/pdf/rbcs/v32n4/a36v32n4. Acesso em: 05, fevereiro, 2015. 